\title{
Sex-Based Differences in the Cytokine Production and Intracellular Signaling Pathways in Patients With Rheumatoid Arthritis
}

\author{
Uday P. PRATAP ${ }^{1} \mathbb{D}$, Lalgi HIMA ${ }^{1} \mathbb{D}$, Thangamani KANNAN $^{1} \mathbb{D}$, Chadrasekaran THYAGARAJAN ${ }^{1} \mathbb{D}$, \\ Hannah P. PRIYANKA ${ }^{1}{ }^{-}$, Ramasamy VASANTHAREKHA ${ }^{1} \mathbb{D}$, Anand PUSHPARANI ${ }^{2}$, \\ Srinivasan THYAGARAJAN ${ }^{1}$ \\ ${ }^{1}$ Integrative Medicine Laboratory, Department of Biotechnology, School of Bioengineering, SRM Institute of Science and Technology, \\ Kattankulathur, Tamil Nadu, India \\ ${ }^{2}$ Department of Anesthesiology, SRM Medical College and Research Center, SRM Institute of Science and Technology, \\ Kattankulathur, Tamil Nadu, India
}

\begin{abstract}
Objectives: This study aims to investigate lymphoproliferation, cytokine production, and intracellular signaling molecules in peripheral blood mononuclear cells (PBMCs) isolated from healthy individuals and rheumatoid arthritis (RA) patients to understand the extent of the involvement of these pathways in the pathogenesis of RA.

Patients and methods: The study included 65 participants (29 males, 36 females; mean age $51.8 \pm 10.3$ years; range, 37 to 71 years) who were categorized into four groups as healthy males ( $n=22$, mean age $49.8 \pm 10.6$ years; range, 39 to 65 years), male RA patients ( $n=7$, mean age $51.8 \pm 13.9$ years; range, 37 to 68 years), healthy females ( $n=20$, mean age $53.7 \pm 8.8$ years; range, 42 to 67 years), and female $R A$ patients ( $n=16$, mean age 52.9 \pm 10.4 years; range, 40 to 71 years). PBMCs were collected from the participants and analyzed for Concanavalin A (Con A)-induced lymphoproliferation using 3-(4,5-dimethylthiazol-2-yl)-2,5-diphenyltetrazolium bromide assay, cytokine production, and phospho-signal transducer and activator of transcription 3 (p-STAT-3), phospho-extracellular-signal-regulated kinase (p-ERK), phospho-cAMP response element binding (p-CREB), and phospho-protein kinase B expressions using enzyme-linked immunosorbent assay. Short form of the Arthritis Impact Measurement Scales 2 and multidimensional health assessment questionnaire were used to measure the level of disability and the quality of life.

Results: In RA patients, production of Con A-induced interleukin (IL)-2 and IL-17 was higher in both sexes while interferon-gamma levels decreased in RA females alone. Expression of p-STAT-3 in PBMCs increased in RA males while it was unaltered in RA females. $\mathrm{p}$-ERK expression was not altered while $p$-CREB expression was enhanced in RA males and females. Protein-protein interaction analyses demonstrated that these and other key signaling molecules were dysregulated in RA patients.

Conclusion: Our results suggest that sex-based differences in RA pathogenesis result from differential alterations in signaling pathways to influence the inflammatory process.

Keywords: Autoimmunity, cytokines, inflammation, phospho-cAMP response element binding, signal transducer and activator of transcription 3.
\end{abstract}

Rheumatoid arthritis (RA) is a chronic inflammatory autoimmune disease predominantly afflicting females characterized by the inflammation of synovial joints with a major impact on physical and psychological health of patients. ${ }^{1-3}$ Autoimmune diseases have been shown to disproportionately affect females than males, with females making up more than $75 \%$ of all cases of RA. ${ }^{4}$ It has been shown that sex disparity affects the impact on course of RA disease, response to treatment, and overall prognosis. ${ }^{5}$ Hormones, genetic factors and clinical

Received: March 07, 2019 Accepted: November 26, 2019 Published online: April 27, 2020

Correspondence: Srinivasan ThyagaRajan, B.V.Sc., PhD. Integrative Medicine Laboratory, Department of Biotechnology School of Bioengineering SRM Institute of Science and Technology, Kattankulathur 603203, Tamil Nadu, India. Tel: 91-9940201794 e-mail: thyagarajan.s@ktr.srmuniv.ac.in

Pratap U, Hima L, Kannan T, Thyagarajan C, Priyanka H, Vasantharekha R, et al. Sex-Based Differences in the Cytokine Production and Intracellular Signaling Pathways in Patients With Rheumatoid Arthritis. Arch Rheumatol 2020;35(4):545-557. 
phenotypes differentially regulate the RA disease. Besides these factors, another possible reason for the sex-based difference in RA has been ascribed to the role of sex hormones. Estrogen and other steroid hormones play a crucial role in the increased incidence of autoimmune diseases that may be related to their robust cellular and humoral immune responses in females than males. ${ }^{6}$ Perhaps, the changes in endocrine functions, particularly the steroid hormones, occurring during the menopausal transition may promote the development of RA.7 Intriguingly, disease activity is ameliorated in 75\% of RA females during pregnancy, which exacerbates after delivery in majority of RA females. ${ }^{8}$

Cell-mediated immunity through $\mathrm{T}$ cell functions influences the pathogenesis of RA. Besides the differences in $\mathrm{T}$ helper 1 (Th1)/Th2 cytokine responses, excessive production of proinflammatory cytokines, such as interleukin (IL)-6, tumor necrosis factor-alpha (TNF- $\alpha$ ), and IL-1 beta contribute to the RA pathogenesis by exacerbating the destruction of joints in these patients. ${ }^{9,10}$ Recently, IL-17 was found to modulate the disease progression and has been suggested as a target therapy for the treatment of RA. ${ }^{10,11}$ There are several functions attributed to IL-17 in the promotion and progression of RA which include angiogenesis, increased production of inflammatory mediators and matrix degrading enzymes, and promotion of osteoclastic bone erosion. ${ }^{12} \mathrm{~A}$ balance in Th1 and Th2 cytokines is a normal phenomenon in healthy individuals while this may be lost in pathogenesis of RA because both Th1 and Th2 cytokines suppress the differentiation of Th17 cells. ${ }^{13}$

Cytokines signal through the intracellular Janus kinase (JAK)/signal transducer and activator of transcription (STAT) pathway during the differentiation of naive $\mathrm{T}$ cells to subsets of $\mathrm{T}$ cells. Dysregulation in JAK/STAT intracellular signaling and the cross talk between JAK/ STAT and the mitogen-activated protein kinase (MAPK) and phosphoinositide 3-kinase (PI-3K)/ protein kinase $\mathrm{B}$ (Akt) pathways play a critical role in RA. ${ }^{14}$ The cross-regulatory and modulatory extracellular-signal-regulated kinase (ERK), cAMP response element binding (CREB), and Akt cell signaling pathways in lymphocytes at the molecular level influence the disease progression through a number of molecules involving neuroendocrine-immune system. Although little is known about the role of STAT-3 phosphorylation level in peripheral blood mononuclear cells (PBMCs) of RA patients, it exerts a key role in the etiology of RA due to its importance in the regulation of Th17 and regulatory T cells. ${ }^{15,16}$

The synergy between cytokines produced by T cells, particularly IL-17, and proinflammatory cytokines play a crucial role in the progression of RA. ${ }^{17}$ Understanding these interactions may be useful in delineating molecular signaling pathways for effective development of therapeutic strategies in RA. Moreover, the literature survey suggests that there is limited evidence for the effect of sex-based differences in the pathogenesis of RA. Differences between males and females in hormone production, physiologic characteristics, chromosome complement, sex-based roles, and behavioral expectations have all been proposed as contributing factors. ${ }^{18}$ To answer these questions, we have examined the effects of sex-based differences on RA pathogenesis based upon the immune profile and cell signaling pathways. We hypothesized that sex-based disparity in disease pathogenesis in RA patients may involve differential regulation of cytokine production and intracellular signaling pathways. Therefore, in this study, we aimed to investigate lymphoproliferation, cytokine production, and intracellular signaling molecules in PBMCs isolated from healthy individuals and RA patients to understand the extent of the involvement of these pathways in the pathogenesis of RA.

\section{PATIENTS AND METHODS}

This study was conducted at the SRM University between September 2014 and March 2016. The study included 65 participants $(29$ males, 36 females; mean age $51.8 \pm 10.3$ years; range, 37 to 71 years) who were categorized into four groups as healthy males $(n=22$, mean age $49.8 \pm 10.6$ years; range, 39 to 65 years), male RA patients $(n=7$, mean age $51.8 \pm 13.9$ years; range, 37 to 68 years), healthy females ( $n=20$, mean age $53.7 \pm 8.8$ years; range, 42 to 67 years), and female RA patients ( $\mathrm{n}=16$, mean age $52.9 \pm 10.4$ years; range, 40 to 71 years). A convenient sample size was chosen to conduct the 
study. A total of 45 subjects with RA symptoms were examined and surveyed by questionnaires and only 23 diagnosed as RA were included. The study protocol was approved by the SRM University Ethics Committee (Human Ethical Committee Approval Number: 559/IEC/2014). A written informed consent was obtained from each participant. The study was conducted in accordance with the principles of the Declaration of Helsinki.

Prior to enrollment, all participants' health status was verified by asking questions regarding their age, height, weight, general health, family history, habits like alcohol or smoking, endocrinological, and neurological diseases. In females, additional information regarding their gynecological status, menstrual abnormalities, and use of contraceptive medications, sleeping patterns, and psychiatric disorders were collected. Females who were under-weight, obese or diabetic, had given birth within the past six months, had recent radiological or ultrasound exams, using oral contraceptives, had gynecological problems (bleeding between periods, vaginal infection, abnormal vaginal bleeding, and endometriosis) and hormone treatments were excluded. Patients with clinical symptoms including joint pains, stiffness, swelling, tenderness, pain and limitation on motion as per American College of Rheumatology (ACR)/European League Against Rheumatism (EULAR) 2010 classification criteria were included. ${ }^{19}$ Patients with alcohol or smoking habits, endocrinological, neurological, and gynecological problems were excluded. Further, they were evaluated through the short form of the Arthritis Impact Measurement Scales 2 (AIMS2-SF) and multidimensional health assessment questionnaire (MDHAQ). The AIMS2-SF measures the individual's functional, social, emotional and physical status through 26 items covering information in five areas of health (physical, symptom, affect, social, and work). ${ }^{20}$ MDHAQ was used to examine the role of physical function, pain, patient global estimate, disease activity index, review of system, and routine assessment of patient index data 3 (RAPID3).

Subjects were briefly interviewed prior to the collection of blood samples between 08:00 a.m. and 10:00 a.m. at the department of hematology at our university's hospital. The whole blood was collected aseptically from healthy controls and RA patients in ethylenediaminetetraacetic acid (EDTA)-coated vacutainers. The PBMCs were isolated from the whole blood using density gradient centrifugation as described previously. ${ }^{21,22}$ In brief, PBMCs were removed from the interface between the Hank's balanced salt solution (HBSS) and the Histopaque, centrifuged, and resulting cells pellet was washed three times in HBSS. After the final wash, cells were resuspended to the desired concentration in complete Roswell Park Memorial Institute-1640 medium supplemented with 5\% fetal calf serum (Sigma-Aldrich, St. Louis, MO, USA) and cultured in vitro.

Peripheral blood mononuclear cells $\left(2 \times 10^{5}\right.$ cells $\left./ \mathrm{mL}\right)$ cultured in triplicate were co-incubated with $0.5,1.25$, and $5 \mu \mathrm{g} / \mathrm{mL}$ of Concanavalin A (Con A) in 96-well and 24-well flat-bottom tissue culture plates. Con A-induced lymphocytes proliferation was performed using 3-(4,5-dimethylthiazol-2-yl)-2,5diphenyltetrazolium bromide (MTT) reagent. ${ }^{22}$ Briefly, cells in 96 well plates were treated with MTT reagent, incubated for three hours and read at $620 \mathrm{~nm}$ after completely solubilizing formazan adduct in isopropanol comprising 37\% hydrochloric acid.

To measure cytokine production, after 24 hours of co-incubation with $1.25 \mu \mathrm{g} / \mathrm{mL}$ of Con A, supernatants were collected for the estimation of cytokine (IL-17, IL-2, and interferongamma [IFN- $\gamma$ ]) production using enzyme-linked immunosorbent assay (ELISA) kits (R\&D Systems, Minneapolis, MN, USA).

Anti-cyclic citrullinated peptide (anti-CCP) level was measured in serum samples using ELISA kits (Cusabio Biotech Co. Ltd., Wuhan, China). Serum was isolated from the non-EDTA-coated vacutainers and analyzed for cortisol assay using ELISA kits (R\&D Systems, Minneapolis, MN, USA).

Cells were lysed in radioimmunoprecipitation assay buffer to analyze the expression of ERK, (phospo-) p-ERK, CREB, p-CREB, Akt, and p-Akt using ELISA (R\&D Systems, Minneapolis, MN, USA) kits. ${ }^{22}$ Briefly, 96-well Corning Costar MaxiSorp ELISA plates were coated with $0.2 \mu \mathrm{g} / \mathrm{mL}$ of primary antibody and incubated overnight at $37^{\circ} \mathrm{C}$. Next day, plates were washed with phosphate-buffered saline and blocked in 
bovine serum albumin blocking buffer for two hours. After two hours of incubation, plates were washed again and incubated with $100 \mu \mathrm{L}$ of sample or respective standards for two hours at room temperature. After two hours of incubation, plates were washed and treated with suitable detection antibody for one hour, followed by horseradish peroxidase (HRP)-tagged secondary antibody and 33,3',5,5'-tetramethylbenzidine (TMB) substrate for the quantification of TMB-HRP colorimetric reaction. Twenty minutes after substrate addition, $2 \mathrm{~N}$ sulphuric acid was added to stop the reaction and the plates were read at $450 \mathrm{~nm}$ using microplate reader.

The STAT-3 [pY705] phosphorylation level was measured in cell lysates samples using ELISA kit as per the kit protocol (ThermoFisher Scientific, Waltham, MA, USA).

Protein interaction databases [Biological General Repository for Interaction Datasets (BIOGRID), IntAct molecular interaction database (IntAct), Molecular INTeraction database (MINT), Database of Interacting Proteins (DIP), Biomolecular Interaction
Network Database (BIND), and Human Protein Reference Database (HPRD)] were used to retrieve the interacting proteins of RA. All protein-protein interaction (PPI) network was visualized using Cytoscape software (version 3.0) (https://cytoscape.org/). Functional enrichment analysis was carried out for the identified clusters using the Database for Annotation, Visualization and Integrated Discovery (DAvID) tool to understand the functional association of clusters in the network. In DAvID tool, enrichment $\mathrm{p}$ value and a false discovery rate (FDR) were calculated by DAvID for each Go term. The Go terms with FDR $<0.05$ were considered as statistically significant.

\section{Statistical analysis}

Differences between groups were measured by Kruskal-Wallis analysis of variance (ANOVA) using GraphPad Prism version 6.0 software (GraphPad Software Inc., San Diego, CA, USA). All values were expressed as mean \pm standard error of the mean. Pearson correlation coefficients were used to examine the potential association between the variables.

Table 1. Demographic and clinical enrollment data of study population

\begin{tabular}{|c|c|c|c|c|c|c|c|c|c|c|c|c|}
\hline & \multicolumn{6}{|c|}{ Healthy controls } & \multicolumn{6}{|c|}{ Rheumatoid arthritis } \\
\hline & \multicolumn{3}{|c|}{ Males } & \multicolumn{3}{|c|}{ Females } & \multicolumn{3}{|c|}{ Males } & \multicolumn{3}{|c|}{ Females } \\
\hline & $\mathrm{n}$ & $\%$ & Mean \pm SD & $\mathrm{n}$ & $\%$ & Mean \pm SD & $\mathrm{n}$ & $\%$ & Mean \pm SD & $\mathrm{n}$ & $\%$ & Mean \pm SD \\
\hline Age (year) & & & $49.8 \pm 10.6$ & & & $53.7 \pm 8.8$ & & & $51.8 \pm 13.9$ & & & $52.9 \pm 10.4$ \\
\hline Number & 22 & & & 20 & & & 7 & & & 16 & & \\
\hline Weight (kg) & & & $66.8 \pm 10.6$ & & & $61.5 \pm 9.3$ & & & $62.8 \pm 7.4$ & & & $62.1 \pm 13.7$ \\
\hline Sex $(\%)$ & & 33.84 & & & 30.79 & & & 10.76 & & & 24.61 & \\
\hline \multicolumn{13}{|l|}{ Diseases } \\
\hline Diabetes mellitus & & 18.18 & & & 15.00 & & & 14.28 & & & 12.50 & \\
\hline Hypertension & & 0 & & & 20.00 & & & 0 & & & 3.00 & \\
\hline \multicolumn{13}{|l|}{ Education } \\
\hline University & & 50.00 & & & 35.00 & & & 40.00 & & & 14.00 & \\
\hline School (1 $1^{\text {st}}-12^{\text {th }}$ grades $)$ & & 50.00 & & & 60.00 & & & 60.00 & & & 76.00 & \\
\hline Uneducated & & 0 & & & 5.00 & & & 0 & & & 20.00 & \\
\hline \multicolumn{13}{|l|}{ Residency } \\
\hline Rural & & 50.00 & & & 10.00 & & & 42.8 & & & 37.5 & \\
\hline Urban & & 50.00 & & & 90.00 & & & 57.2 & & & 62.5 & \\
\hline \multicolumn{13}{|l|}{$\begin{array}{l}\text { Physical condition } \\
\text { of the joints }\end{array}$} \\
\hline Tender joints (no.) & & 0.00 & & & 0.00 & & & 6.00 & & & 12.00 & \\
\hline Swollen Joints (no.) & & 0.00 & & & 0.00 & & & 17.00 & & & 12.00 & \\
\hline
\end{tabular}




\section{RESULTS}

A survey of the participants enrolled in the study provided the demographic and clinical characteristics of the study population (Table 1). Healthy individuals showed no significant differences in the weight profiles compared to RA groups. Among the survey population, 33.84\% were healthy males, $30.79 \%$ healthy females, $10.76 \%$ RA males and $24.61 \%$ RA females. The distribution of diabetes mellitus was $18.18 \%$ and $15 \%$ in healthy males and females, respectively, while it was $14.28 \%$ and $12.5 \%$ in RA males and RA females, respectively. The prevalence of hypertension in the study population was $20 \%$ in healthy females and 3\% in RA females. Most of the study participants had received education at least up to the school level ( $\left(1^{\text {st }}-12^{\text {th }}\right.$ grades) and most of them were from urban areas.

The AIMS2-SF survey revealed a significant $(p<0.05)$ increase in physical and symptom scores in RA males and females compared to healthy controls (Table 2). Descriptive analysis was used to calculate interquartile ranges and analyzed using Kruskal-Wallis ANOVA. Also, a significant $(p<0.05)$ increase in affect score in RA females was observed in comparison to healthy controls.

Multidimensional health assessment questionnaire scoring of physical function and review of system showed no significant difference in RA patients. An analysis of the questionnaire regarding pain scale and patient global estimate measured at 0-10 scale by visual analog scale to examine the severity of disease in health condition revealed that the RA patients had higher score on pain scale compared to healthy controls. Results from RAPID3 score, which is a sum of the three patient report scores (functional impairment on MDHAQ, pain, and global estimate), demonstrated a very high score $(>12)$ in RA patients, indicating the severity of the disease condition that may predict the subsequent disease progression in RA patients. The work component was not applicable in our study since most of the patients were retired, disabled or unemployed.

Serum anti-CCP levels significantly $(p<0.05)$ increased in both RA males and females compared to healthy controls (Table 2). Serum cortisol was significantly $(p<0.05)$ higher in both RA males $(p<0.05)$ and females $(p<0.001)$ compared to healthy controls (Table 2).

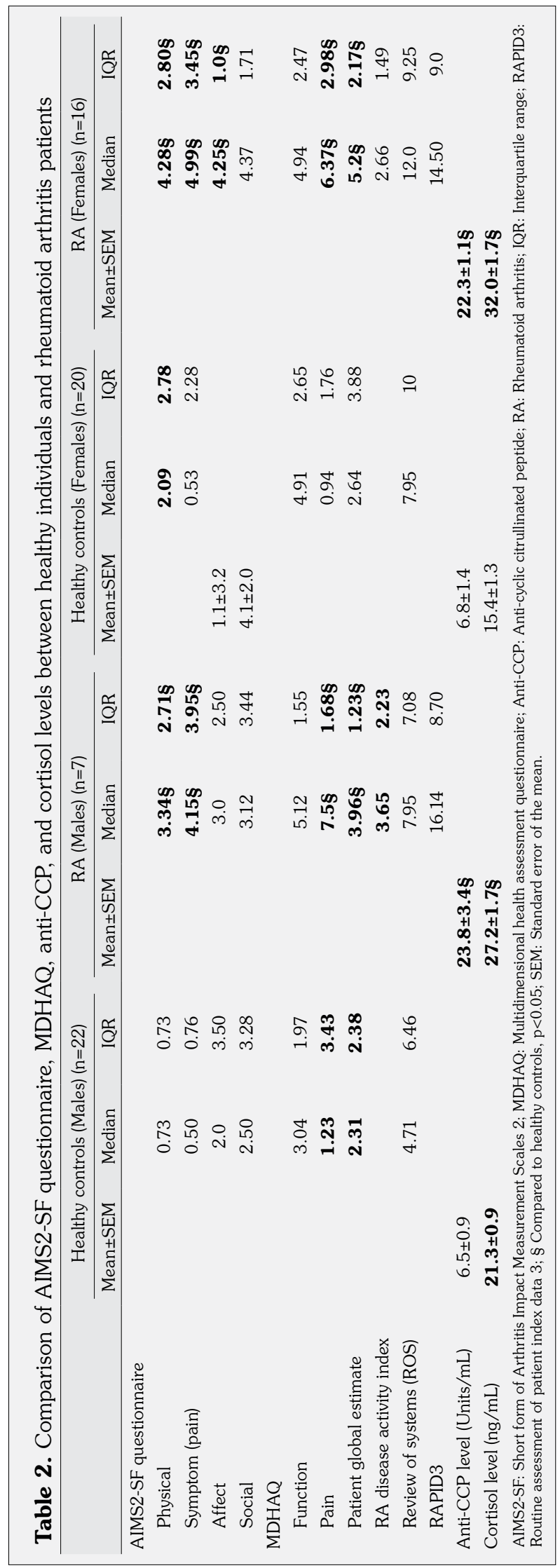


Table 3. Regression analysis of serum cortisol anti-CCP levels, pain score, and cytokine production in RA patients

\begin{tabular}{lcccccc}
\hline Regression analysis & RA males & $p$ & Equation & RA females & $p$ & Equation \\
\hline $\begin{array}{l}\text { Anti-CCP level }(\text { Units } / \mathrm{mL}) \\
\text { vs. serum cortisol }(\mathrm{ng} / \mathrm{mL})\end{array}$ & $\mathrm{R}^{2}=0.404$ & $<0.005$ & $\mathrm{Y}=0.7297^{*} \mathrm{X}+11.66$ & $\mathrm{R}^{2}=0.423$ & $<0.0005$ & $\mathrm{Y}=0.9674 * \mathrm{X}+11.97$ \\
$\begin{array}{l}\text { IL-17 }(\mathrm{pg} / \mathrm{mL}) \text { vs. serum } \\
\text { cortisol }(\mathrm{ng} / \mathrm{mL})\end{array}$ & $\mathrm{R}^{2}=0.378$ & $<0.01$ & $\mathrm{Y}=0.09575 * \mathrm{X}+3.334$ & $\mathrm{R}^{2}=0.691$ & $<0.0001$ & $\mathrm{Y}=0.218^{*} \mathrm{X}-7.886$ \\
\hline
\end{tabular}

Anti-CCP: Anti-cyclic citrullinated peptide; RA: Rheumatoid arthritis; IL: Interleukin; $y=m x+c$; Where $y$ is dependent variable, $m$ is slope, $x$ is independent variable, and $\mathrm{c}$ is intercept for a given line.

Linear regression analysis was performed using $\mathrm{y}=\mathrm{mx}+\mathrm{c}$, where $\mathrm{y}$ is the dependent variable, $\mathrm{m}$ is the slope, $\mathrm{x}$ is the independent variable, and $\mathrm{c}$ is the intercept for a given line. Linear regression analysis revealed a positive correlation between anti-CCP (independent variable) and serum cortisol level (dependent variable) in $\mathrm{RA}$ males (R2=0.404, $\mathrm{p}<0.005)$ and females $(\mathrm{R} 2=0.423, \mathrm{p}<0.0005)$ (Table 3). Similar positive correlation was observed between serum cortisol levels (dependent variable) and IL-17 (independent variable) production in $\mathrm{RA}$ males $(\mathrm{R} 2=0.378$, $\mathrm{p}<0.01)$ and females $(\mathrm{R} 2=0.691, \mathrm{p}<0.0001)$.

No change in lymphocyte proliferation was observed in RA males and females compared to healthy controls (Figure 1a, b). A significant $(p<0.0001)$ increase in IL-2 production was observed in RA males and females (Figure 2a). In contrast, a significant $(p<0.01)$ decrease in
IFN- $\gamma$ production was observed in RA females alone compared to healthy controls (Figure 2b). Similarly, a significant $(p<0.05)$ increase in IL-17 production was evident in both RA males and females compared to healthy controls (Figure 2c).

A significant $(p<0.05)$ increase in RA males alone was observed compared to healthy controls (Figure 3). A significant increase in the expression of Con A-induced $\mathrm{p}$-CREB/total CREB was observed in RA males $(p<0.001)$ and females $(p<0.01)$ compared to healthy controls (Figure $4 \mathrm{a}, \mathrm{b}$ ). The expression of $\mathrm{p}$-Akt/total Akt was decreased in both RA males and females (Figure 4a, b).

To better understand the regulatory mechanism governing immune cell differentiation and signaling pathways, we used protein interaction databases (BIOGRID, IntAct, (a)

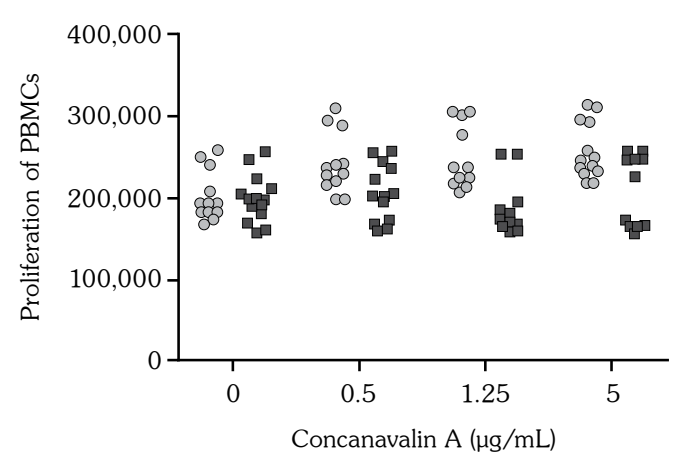

Healthy controls

Rheumatoid arthritis women (b)

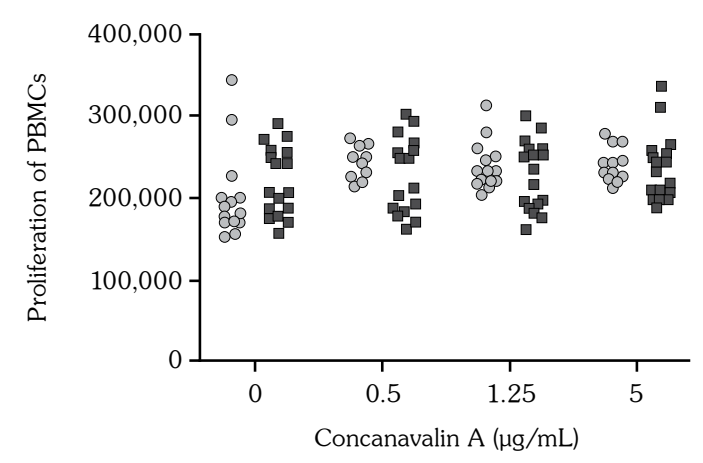

Healthy controls $\square$ Rheumatoid arthritis women

Figure 1. Concanavalin A (Con A)-induced proliferation of peripheral blood mononuclear cells (PBMCs) from males (a) and females (b) of healthy controls and rheumatoid arthritis groups. PBMCs $\left(2 \times 10^{5} \mathrm{cells} / \mathrm{mL}\right)$ were incubated with $0,0.5,1.25$, and $5 \mu \mathrm{g} / \mathrm{mL}$ of Concanavalin A for 72 hours and proliferation was assessed using 3-(4,5-dimethylthiazol-2-yl)-2,5-diphenyltetrazolium bromide assay.

Con A: Concanavalin A; PBMCs: Peripheral blood mononuclear cells. 
(a)

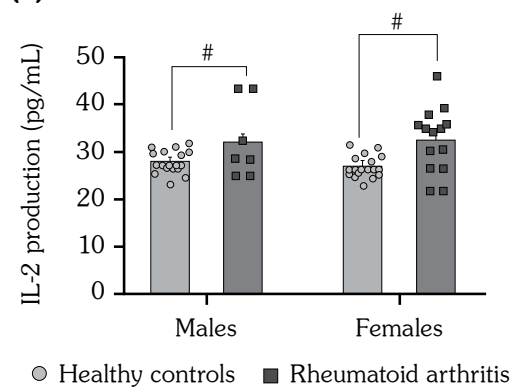

(b)

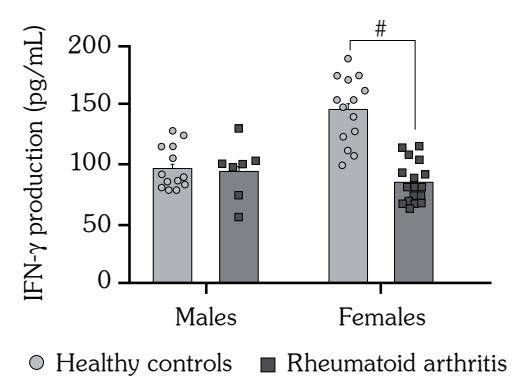

(c)

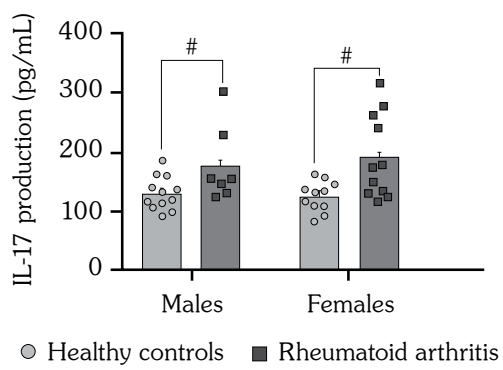

Figure 2. Concanavalin A-induced cytokine production [IL-2 (a), IFN- $\gamma$ (b), and IL-17 (c)] in PBMCs from males and females of healthy controls and rheumatoid arthritis groups. PBMCs $\left(2 \times 10^{5}\right.$ cells/well) were incubated with $1.25 \mu \mathrm{g} / \mathrm{mL}$ of Con A for a period of 24 hours.

IFN- $\gamma$ : Interferon-gamma; IL: Interleukin; \# p<0.05 compared to healthy controls; PBMCs: Peripheral blood mononuclear cells.

MINT, DIP, BIND and HPRD) to predict the putative interacting partners of cytokines and intracellular signaling molecules. Individual PPI network was constructed for RA from the curated list with immediate one neighbor interaction to represent the core mechanism of the disease. The constructed network with multifaceted interaction proteins describes the complex mechanism of interactome. The top hub proteins identified in RA were selected according to the degree of connectivity. Based on the information from STRING database, a total of 294 protein interactions with combined scores $>0.4$ were included in the PPI network

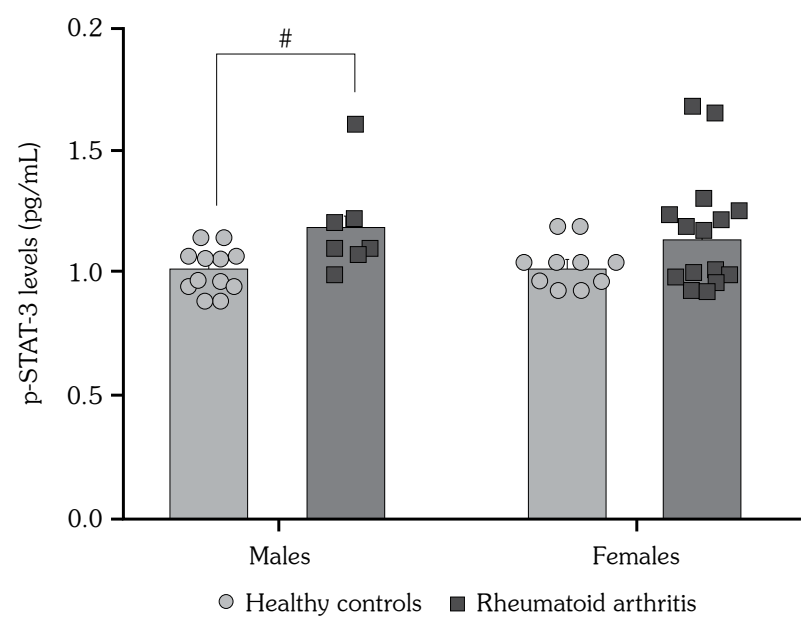

Figure 3. Signal transducer and activator of transcription 3 phosphorylation induced by Concanavalin A in peripheral blood mononuclear cells from males and females of healthy controls and rheumatoid arthritis groups.

p-STAT-3: Phospho-signal transducer and activator of transcription 3; \# $\mathrm{p}<0.05$ compared to healthy controls.
(Supplementary Figure 1). This search resulted in the identification of IFN- $\gamma$, TNF, Akt, IL-6, IL-2, and CREB 1 as some of the key molecules of interactome (Supplementary Figure 2). Therefore, clusters were formed to understand the multifaceted interaction between cytokines and signaling molecules associated with RA. Cluster 1 contains IL-2 and neighboring interacting molecules such as IL2RG, SHC1, NGFR, MAPKAPK2, MAPK14, and AKT1 genes (Figure 5a). Cluster 2 contains CREB1 and neighboring interacting molecules such as CREBBP, SIRT1, HIST1, and HDAC1 genes that regulate histone acetylation (Figure 5b). Similarly, cluster 3 contains TNF and neighboring interacting molecules such as TBK1, TRAF2, TNFRSF1B, CASP8, FADD, and TP53 genes that regulate the nuclear factor-kappa $\mathrm{B}$ (NF- $\mathrm{\kappa B})$ activation and promotes inflammatory response and apoptosis (Figure 5c). Likewise, cluster 4 contains interacting molecules such as TNFRSF1A, MAP3K7, IKBKB, IKBKG, and NFKB1A genes (Figure $5 \mathrm{~d}$ ). Functional analysis of these clusters in RA suggested that dysregulation of signaling events, and cytokine levels were associated with inflammatory response network.

\section{DISCUSSION}

The results from the present study demonstrated sex-based differences in the cytokine production and cellular signaling factors in RA patients implicating the complex role exerted by the immune cells and their secreted 
(a)

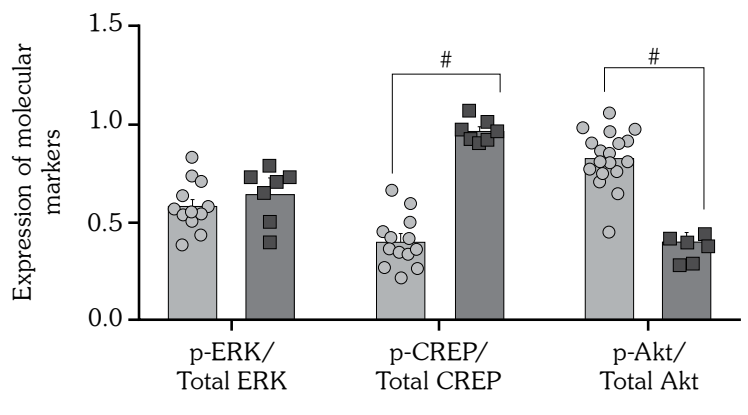

(b)

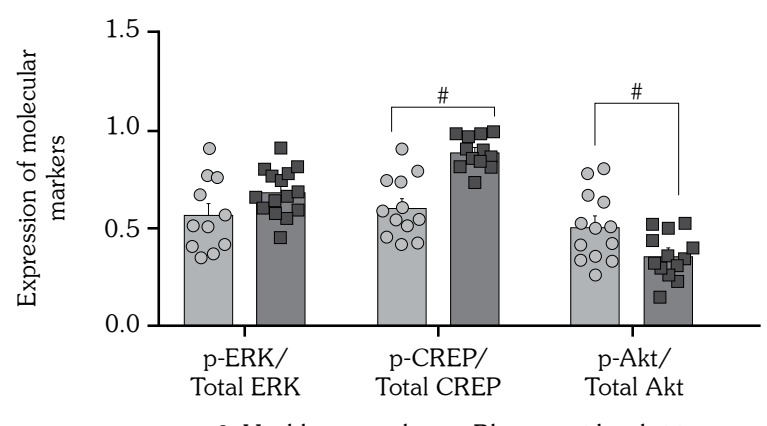

Figure 4. Expression of $\mathrm{p}$-ERK/total ERK, $\mathrm{p}-\mathrm{CREB} / \mathrm{total} \mathrm{CREB}$ and $\mathrm{p}$-Akt/total Akt induced by Concanavalin A in peripheral blood mononuclear cells from males (a) and females (b) of healthy controls and rheumatoid arthritis groups.

ERK: Extracellular-signal-regulated kinase; p: Phospho; CREB: cAMP response element binding; Akt: Protein kinase B; \# $<<0.05$ compared to healthy controls.

products in the disease pathogenesis. Based on the published literature, this is the first type of study investigating sex-based differences in immunomodulation and cell signaling pathways in RA patients to understand disease-associated alterations in parameters examined.

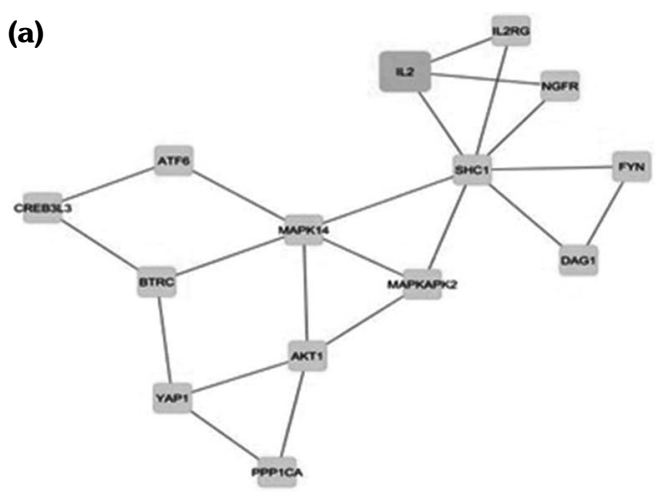

(c)

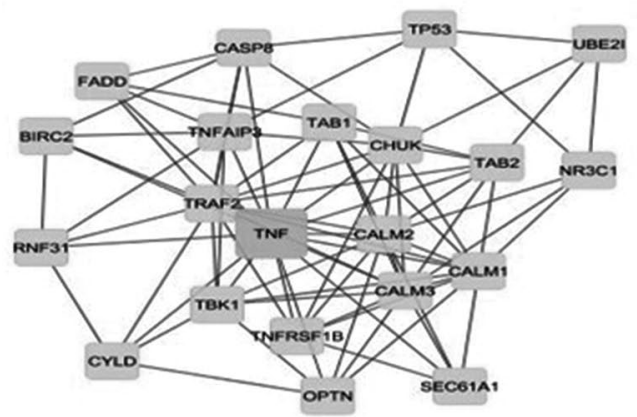

The RA patients had higher scores in physical, symptom, and affect measurement compared to healthy individuals as per AIMS2-SF, while the severity of the disease was established through higher scores in functional, pain and diseases activity index as per MDHAQ-RAPID3. ${ }^{20-23}$

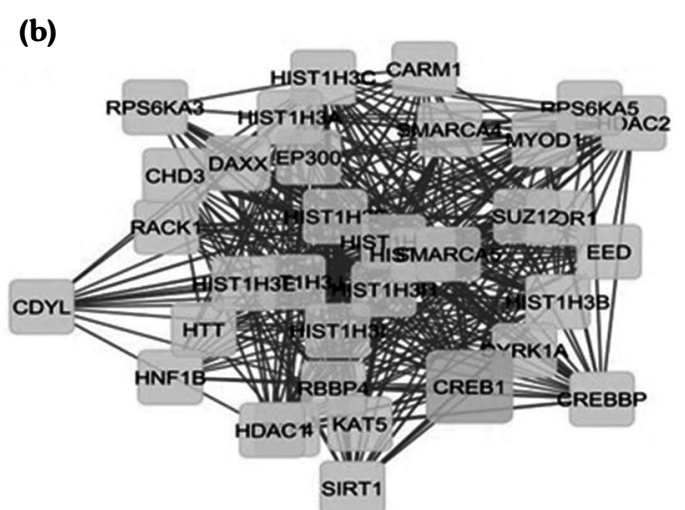

(d)

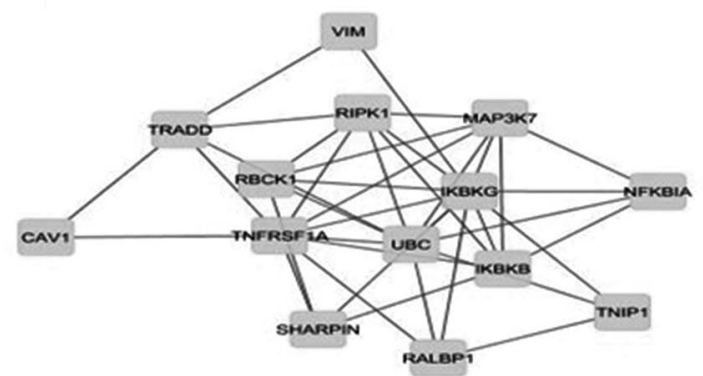

Figure 5. (a-d) Protein-protein interaction network of differentially expressed genes identified for rheumatoid arthritis. 
Sex hormones have been known to markedly influence RA disease pathogenesis differently in males and females. A study suggested that RA disease activity measures were worse in females than males. ${ }^{4}$ Quantitative Patient Questionnaires in Standard Monitoring of Patients with Rheumatoid Arthritis study by Sokka et al. ${ }^{5}$ quantitatively analyzed a large multinational cross-sectional cohort of patients with RA and found that all disease activity measures were higher in the female RA patients than males suggesting that there is a sex-based disparity in RA.

Higher level of serum cortisol reported in RA patients may also be due to chronic inflammation. ${ }^{24}$ Lymphoproliferation was reduced following Con A stimulation of PBMCs in RA patients that may be related to high levels of cortisol observed in the present study as it has been shown to inhibit the proliferation of lymphocytes in both healthy individuals and RA patients. ${ }^{25}$ The higher levels of IL-2 observed in both males and females indicated a predominance of Th1 cytokines that may be associated with a reduced subset of Th1-type cluster of differentiation $4(\mathrm{CD} 4)+\mathrm{T}$ cells producing anti-inflammatory cytokine, IL-10, and thus, promoting the inflammatory processes in RA. ${ }^{26,27}$ However, a significant decrease in IFN- $\gamma$ level, another Th1 cytokine, was observed only in $\mathrm{RA}$ females that may be related to the cytokine's role as a potent activator of macrophages and therefore, TNF- $\alpha$ production. ${ }^{28,29}$ This notion has been supported by the finding that administration of anti-TNF- $\alpha$ antibodies in RA patients increased IFN- $\gamma$-positive CD4+ T cells. ${ }^{30}$ In contrast to IFN- $\gamma$ production, an increase in Con A-induced IL-17 production in both males and females may synergistically activate IL-6, TNF- $\alpha$, and C-reactive protein creating a favorable inflammatory environment in RA patients. ${ }^{17}$ TNF- $\alpha$ plays a key factor in the pathogenesis of RA through activation of STAT3 signaling pathways. ${ }^{31}$ Even though IL-17 levels were higher in both males and females, p-STAT3 expression was upregulated in males alone, suggesting that differential expression of intracellular signaling pathways may regulate its level including various other factors such as Nitric oxide (NO), B-cell lymphoma-2 (Bcl-2), and micro-ribonucleic acid (micro-RNA) to influence the pathogenesis. ${ }^{17}$
Heightened production of proinflammatory cytokines in the presence of high levels of cortisol may have been due to the decreased sensitivity of immune cells to glucocorticoids, which not only exacerbate inflammation in these patients but also promote fatigue and pain. ${ }^{32}$ Furthermore, a decrease in antioxidant enzyme activities (superoxide dismutase, catalase, glutathione peroxidase, and glutathione-stransferase) was observed in PBMCs of RA patients revealing that the disease-associated increase in free radicals may have promoted the progression of the disease through upregulation of proinflammatory cytokines (Supplementary Table 1).

Cell signaling pathways such as MAPKs along with intracellular molecules such as Akt and CREB occupy a central place in mediating the actions of various cytokines, hormones, growth factors, and other ligands through the activation of genes promoting or suppressing inflammation in autoimmune diseases. ${ }^{33}$ Inhibition of increased expression of CREB has improved abnormal synovial cell functions in RA while others have demonstrated that activation and induction of AMP-activated protein kinase/CREB pathway along with manganese-dependent superoxide dismutase inhibited inflammatory processes suggesting that $\mathrm{CREB}$ may have diverse functions in the pathogenesis of RA. ${ }^{34,35}$ CREB is activated by both the mitogen-activated protein kinase kinase (MEK)/ERK and p38 pathways, and upon activation, it regulates the expression of phosphatases to down-regulate MAPK activity. ${ }^{36}$ We reason that lack of alterations in ERK expression in RA patients may have been due to feedback inhibition of MEK/ERK signaling. Akt expression was observed to be lower in both males and females in our study while others have reported it to be highly expressed in RA demonstrating that downregulation of its expression inhibited the production of proinflammatory cytokines and therefore, the severity of the disease. ${ }^{37}$ This divergent finding on Akt expression may be dependent on the patient population and sex of the patients used to examine its levels. Sex-based differences in immune parameters and intracellular signaling pathways in RA males and females in the present study warrant further investigation as these effects may be due to the activation of different 
subset of immune cells and thus, influence immune responses and intracellular signaling pathways.

To further understand the sex-based differences in immune parameters and cell signaling pathways in RA males and females, PPI network analysis was used to predict the link between genes using a cluster analysis. A grid layout plotted to connect the dysregulated cytokines and signaling molecules identified in PBMCs from RA patients using PPI network analysis revealed key interactomes involving IL-2, CREB, and TNF. Interaction of IL-2 with neighboring interacting molecules regulates a number of cellular processes including stress and inflammatory responses, regulation of gene expression, and cell proliferation. ${ }^{38-40}$ Interaction of CREB1 with other molecules such as CREBBP, SIRT1, HIST1, and HDAC1 suggests that SIRT1 may upregulate the production of proinflammatory cytokines in RA synovial fibroblasts. ${ }^{41}$ We further examined the involvement of other proinflammatory cytokines such as TNF- $\alpha$ and the associated molecules involved in the Th17 cell differentiation using protein interaction database to predict the putative interacting partners of TNF- $\alpha$. This search resulted in the identification of TBK1, FADD, TRAF2, TAB1, TNFRSF1B, and CASP8 as components of TNF- $\alpha$ interactome. TNF- $\alpha$ has been reported to enhance the effect of IL-17A on secretion of proinflammatory cytokines and chemokines by rheumatoid synoviocytes. ${ }^{42}$ Importantly, these components regulate the $\mathrm{NF}-\kappa \mathrm{B}$ activation and regulate proliferation, apoptosis, and inflammatory response. ${ }^{43}$

This study has some limitations such as the number of RA patients, unequal distribution of male and female RA patients, and unavailability of data on the type of drugs and disease duration. However, future studies should be conducted improving the sample size and examining the role of different subsets of immune cells and the dysregulated neuroendocrine-immune interactions in the RA patients.

In conclusion, the present study demonstrated that RA disease pathogenesis may be dependent on the sex-based differences in immunomodulation influenced by multiple pathways involving a number of intracellular molecules. Findings from our study may provide further insight to clinicians to increase the accuracy of predictions while treating male and female patients with RA.

\section{Acknowledgements}

We thank all the participants who volunteered in the study, Dr. T. Swarnalingam, Tagore Medical College, Tamil Nadu and all the students in our laboratory for their assistance in recruiting volunteers for blood collection.

\section{Declaration of conflicting interests}

The authors declared no conflicts of interest with respect to the authorship and/or publication of this article.

\section{Funding}

The study was supported by the Department of Biotechnology, SRM Institute of Science and Technology, Kattankulathur, TN, India.

\section{REFERENCES}

1. González DA, Díaz BB, Rodríguez Pérez Mdel C, Hernández AG, Chico BN, de León AC. Sex hormones and autoimmunity. Immunol Lett 2010;133:6-13.

2. Sturgeon JA, Finan PH, Zautra AJ. Affective disturbance in rheumatoid arthritis: psychological and disease-related pathways. Nat Rev Rheumatol 2016;12:532-42.

3. Scott DL, Smith C, Kingsley G. Joint damage and disability in rheumatoid arthritis: an updated systematic review. Clin Exp Rheumatol 2003;21:S20-7.

4. van Vollenhoven RF. Sex differences in rheumatoid arthritis: more than meets the eye... BMC Med 2009;7:12.

5. Sokka T, Toloza S, Cutolo M, Kautiainen H, Makinen H, Gogus F, et al. Women, men, and rheumatoid arthritis: analyses of disease activity, disease characteristics, and treatments in the QUEST-RA study. Arthritis Res Ther 2009;11:R7.

6. Ansar Ahmed S, Penhale WJ, Talal N. Sex hormones, immune responses, and autoimmune diseases. Mechanisms of sex hormone action. Am J Pathol 1985;121:531-51.

7. Bove R. Autoimmune diseases and reproductive aging. Clin Immunol 2013;149:251-64.

8. Oka M. Effect of pregnancy on the onset and course of rheumatoid arthritis. Ann Rheum Dis 1953;12:227-9.

9. Brennan FM, McInnes IB. Evidence that cytokines play a role in rheumatoid arthritis. J Clin Invest 2008;118:3537-45.

10. Lubberts $\mathrm{E}$. Role of T lymphocytes in the development of rheumatoid arthritis. Implications for treatment. Curr Pharm Des 2015;21:142-6. 
11. Samson M, Audia S, Janikashvili N, Ciudad M, Trad $\mathrm{M}$, Fraszczak $\mathrm{J}$, et al. Brief report: inhibition of interleukin- 6 function corrects Th17/Treg cell imbalance in patients with rheumatoid arthritis. Arthritis Rheum 2012;64:2499-503.

12. Roeleveld DM, Koenders MI. The role of the Th17 cytokines IL-17 and IL-22 in Rheumatoid Arthritis pathogenesis and developments in cytokine immunotherapy. Cytokine 2015;74:101-7.

13. Park H, Li Z, Yang XO, Chang SH, Nurieva R, Wang $\mathrm{YH}$, et al. A distinct lineage of CD4 $\mathrm{T}$ cells regulates tissue inflammation by producing interleukin 17. Nat Immunol 2005;6:1133-41.

14. Malemud CJ. Intracellular signaling pathways in rheumatoid arthritis. J Clin Cell Immunol 2013;4:160.

15. Park JS, Kwok SK, Lim MA, Kim EK, Ryu JG, Kim SM, et al. STA-21, a promising STAT-3 inhibitor that reciprocally regulates Th17 and Treg cells, inhibits osteoclastogenesis in mice and humans and alleviates autoimmune inflammation in an experimental model of rheumatoid arthritis. Arthritis Rheumatol 2014;66:918-29.

16. Ju JH, Heo YJ, Cho ML, Jhun JY, Park JS, Lee SY, et al. Modulation of STAT-3 in rheumatoid synovial $\mathrm{T}$ cells suppresses Th17 differentiation and increases the proportion of Treg cells. Arthritis Rheum 2012;64:3543-52.

17. Konya C, Paz Z, Apostolidis SA, Tsokos GC. Update on the role of Interleukin 17 in rheumatologic autoimmune diseases. Cytokine 2015;75:207-15.

18. Ortona E, Pierdominici M, Maselli A, Veroni C, Aloisi F, Shoenfeld Y. Sex-based differences in autoimmune diseases. Ann Ist Super Sanita 2016;52:205-12.

19. Aletaha D, Neogi T, Silman AJ, Funovits J, Felson DT, Bingham CO 3rd, et al. 2010 rheumatoid arthritis classification criteria: an American College of Rheumatology/European League Against Rheumatism collaborative initiative. Ann Rheum Dis 2010;69:1580-8.

20. Guillemin F, Coste J, Pouchot J, Ghézail M, Bregeon C, Sany J. The AIMS2-SF: a short form of the Arthritis Impact Measurement Scales 2. French Quality of Life in Rheumatology Group. Arthritis Rheum 1997;40:1267-74.

21. ThyagaRajan S, Madden KS, Kalvass JC, Dimitrova SS, Felten SY, Felten DL. L-deprenyl-induced increase in IL-2 and NK cell activity accompanies restoration of noradrenergic nerve fibers in the spleens of old F344 rats. J Neuroimmunol 1998;92:9-21.

22. Priyanka HP, Sharma U, Gopinath S, Sharma V, Hima L, ThyagaRajan S. Menstrual cycle and reproductive aging alters immune reactivity, NGF expression, antioxidant enzyme activities, and intracellular signaling pathways in the peripheral blood mononuclear cells of healthy women. Brain Behav Immun 2013;32:131-43.

23. Pincus T, Sokka T. Can a Multi-Dimensional Health Assessment Questionnaire (MDHAQ) and Routine Assessment of Patient Index Data (RAPID) scores be informative in patients with all rheumatic diseases? Best Pract Res Clin Rheumatol 2007;21:733-53.
24. Straub RH. Interaction of the endocrine system with inflammation: a function of energy and volume regulation. Arthritis Res Ther 2014;16:203.

25. van den Brink HR, van Wijk MJ, Bijlsma JW. Influence of steroid hormones on proliferation of peripheral blood mononuclear cells in patients with rheumatoid arthritis. Br J Rheumatol 1992;31:663-7.

26. Gerli R, Bistoni O, Russano A, Fiorucci S, Borgato $\mathrm{L}$, Cesarotti ME, et al. In vivo activated $\mathrm{T}$ cells in rheumatoid synovitis. Analysis of Th1- and Th2-type cytokine production at clonal level in different stages of disease. Clin Exp Immunol 2002;129:549-55.

27. Yudoh K, Matsuno H, Nakazawa F, Yonezawa T, Kimura T. Reduced expression of the regulatory CD4+ $\mathrm{T}$ cell subset is related to Th1/Th2 balance and disease severity in rheumatoid arthritis. Arthritis Rheum 2000;43:617-27.

28. Philip R, Epstein LB. Tumour necrosis factor as immunomodulator and mediator of monocyte cytotoxicity induced by itself, gamma-interferon and interleukin-1. Nature 1986;323:86-9.

29. Corradin SB, Buchmüller-Rouiller Y, Mauël J. Phagocytosis enhances murine macrophage activation by interferon-gamma and tumor necrosis factor-alpha. Eur J Immunol 1991;21:2553-8.

30. Schuerwegh AJ, Van Offel JF, Stevens WJ, Bridts $\mathrm{CH}$, De Clerck LS. Influence of therapy with chimeric monoclonal tumour necrosis factor-alpha antibodies on intracellular cytokine profiles of $\mathrm{T}$ lymphocytes and monocytes in rheumatoid arthritis patients. Rheumatology (Oxford) 2003;42:541-8.

31. Hanlon MM, Rakovich T, Cunningham CC, Ansboro $\mathrm{S}$, Veale DJ, Fearon U, et al. STAT3 Mediates the Differential Effects of Oncostatin M and TNF $\alpha$ on RA Synovial Fibroblast and Endothelial Cell Function. Front Immunol 2019;10:2056.

32. Davis MC, Zautra AJ, Younger J, Motivala SJ, Attrep J, Irwin MR. Chronic stress and regulation of cellular markers of inflammation in rheumatoid arthritis: implications for fatigue. Brain Behav Immun 2008;22:24-32.

33. Thalhamer T, McGrath MA, Harnett MM. MAPKs and their relevance to arthritis and inflammation. Rheumatology (Oxford) 2008;47:409-14.

34. Takeba Y, Suzuki N, Wakisaka S, Takeno M, Kaneko $\mathrm{A}$, Asai $\mathrm{T}$, et al. Involvement of cAMP responsive element binding protein (CREB) in the synovial cell hyperfunction in patients with rheumatoid arthritis. Clin Exp Rheumatol 2000;18:47-55.

35. Thornton CC, Al-Rashed F, Calay D, Birdsey $\mathrm{GM}$, Bauer $\mathrm{A}$, Mylroie $\mathrm{H}$, et al. Methotrexatemediated activation of an AMPK-CREB-dependent pathway: a novel mechanism for vascular protection in chronic systemic inflammation. Ann Rheum Dis 2016;75:439-48

36. Casals-Casas C, Alvarez E, Serra M, de la Torre C, Farrera C, Sánchez-Tilló E, et al. CREB and AP-1 activation regulates MKP-1 induction by LPS or $\mathrm{M}-\mathrm{CSF}$ and their kinetics correlate with macrophage activation versus proliferation. Eur $\mathrm{J}$ Immunol 2009;39:1902-13. 
37. Wu T, Mohan $\mathrm{C}$. The AKT axis as a therapeutic target in autoimmune diseases. Endocr Metab Immune Disord Drug Targets 2009;9:145-50.

38. Zhang W, Liu HT. MAPK signal pathways in the regulation of cell proliferation in mammalian cells. Cell Res 2002;12:9-18.

39. Wen AY, Sakamoto KM, Miller LS. The role of the transcription factor CREB in immune function. J Immunol 2010;185:6413-9.

40. Straub RH. Rheumatoid arthritis: Stress in RA: a trigger of proinflammatory pathways? Nat Rev Rheumatol 2014;10:516-8.

41. Niederer F, Ospelt C, Brentano F, Hottiger MO, Gay RE,
Gay S, et al. SIRT1 overexpression in the rheumatoid arthritis synovium contributes to proinflammatory cytokine production and apoptosis resistance. Ann Rheum Dis 2011;70:1866-73.

42. Robert M, Miossec, P. IL-17 in Rheumatoid Arthritis and Precision Medicine: From Synovitis Expression to Circulating Bioactive Levels. Front Med (Lausanne) 2018;5:364

43. Raghav SK, Gupta B, Agrawal C, Chaturvedi VP, Das HR. Expression of TNF-alpha and related signaling molecules in the peripheral blood mononuclear cells of rheumatoid arthritis patients. Mediators Inflamm 2006;2006:12682.

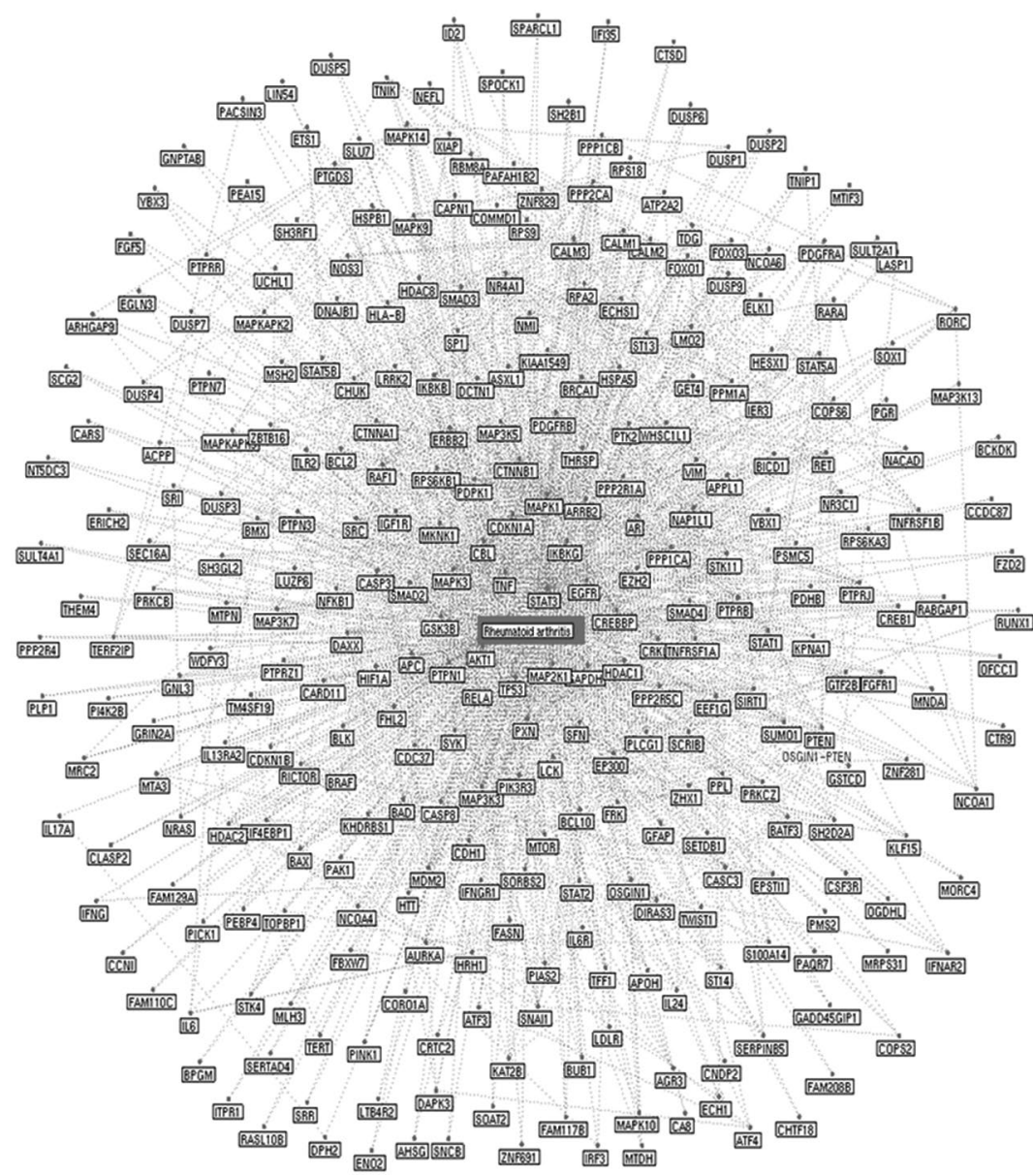

Supplementary Figure 1. STRING database of a total of 294 protein interactions in rheumatoid arthritis. 


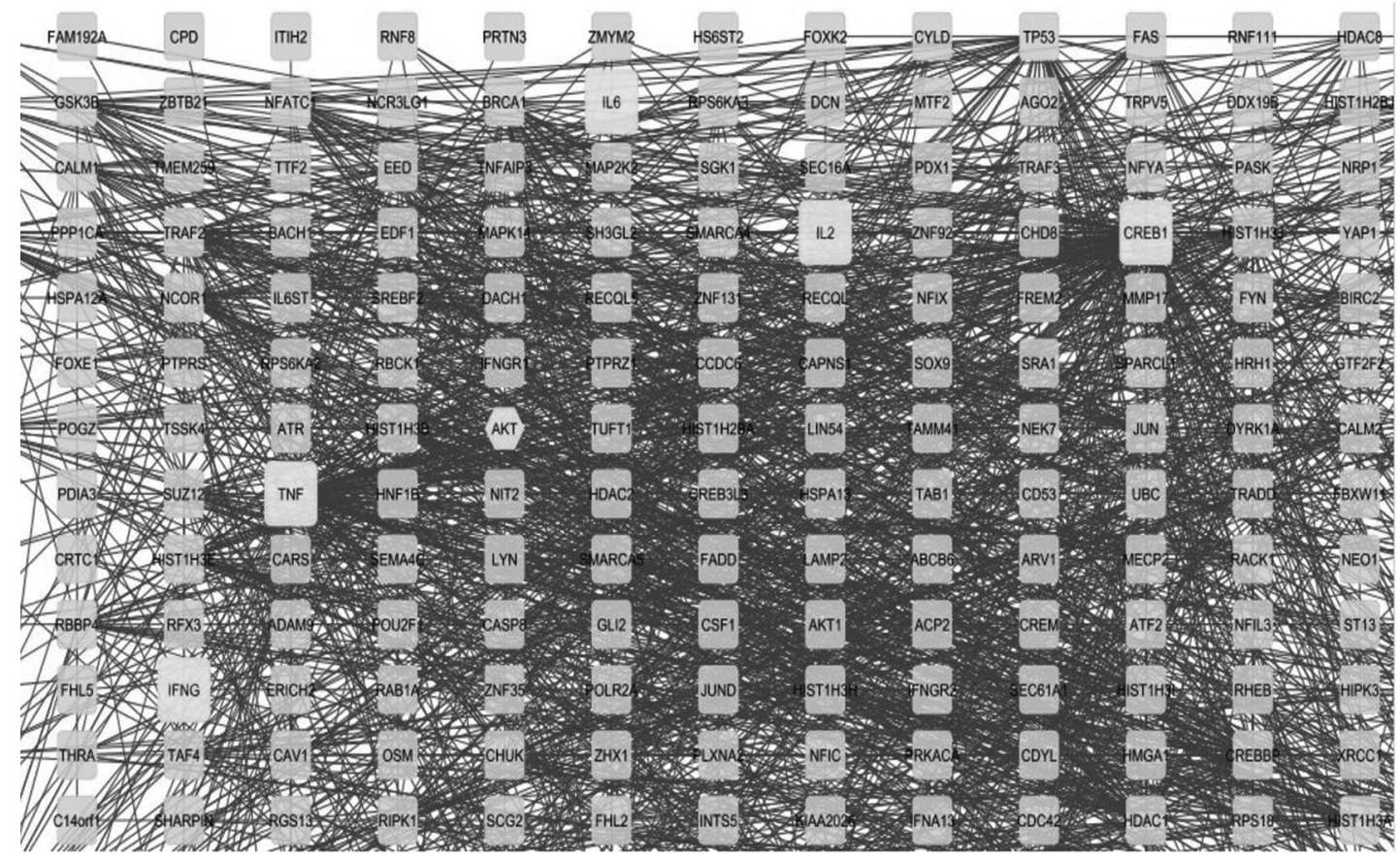

Supplementary Figure 2. A grid layout of gene modules identified for rheumatoid arthritis (RA). Network view of RA interactome is shown as grid layout. Molecules that are involved in disease progression identified in our study are shown in yellow color while closely associated molecules are in blue color.

Supplementary Table 1. Antioxidant enzymes activity in male and female healthy participants and those with rheumatoid arthritis

\begin{tabular}{|c|c|c|c|c|}
\hline & SOD & CAT & GPx & GST \\
\hline Groups & Mean \pm SEM & Mean \pm SEM & Mean \pm SEM & Mean \pm SEM \\
\hline Healthy control (males) & $8.4 \pm 0.2$ & $116.2 \pm 8.6$ & $3.5 \pm 0.3$ & $1.3 \pm 0.1$ \\
\hline Rheumatoid arthritis (males) & $5.9 \pm 0.5^{*}$ & $42.0 \pm 5.2^{*}$ & $0.5 \pm 0.1^{*}$ & $1.2 \pm 0.2$ \\
\hline Healthy control (females) & $11.6 \pm 0.2$ & $155.1 \pm 13.8$ & $0.9 \pm 0.1$ & $1.5 \pm 0.1$ \\
\hline Rheumatoid arthritis (females) & $6.5 \pm 0.3^{*}$ & $71.2 \pm 6.0^{*}$ & $0.3 \pm 0.0^{*}$ & $1.2 \pm 0.1^{*}$ \\
\hline
\end{tabular}

SOD: Superoxide dismutase; CAT: Catalase; GPx: Glutathione peroxidase; GST: Glutathione-S- transferase; SEM: Standard error of the mean; * $\mathrm{p}<0.05$ compared to healthy controls. 